\author{
Nota Científica \\ (Short Communication)
}

\title{
ALIMENTOS CONSUMIDOS POR LA ARDILLA ENDÉMICA DE PEROTE (XEROSPERMOPHILUS PEROTENSIS) EN EL VALLE DE PEROTE, VERACRUZ, MÉXICO
}

\author{
FOOD CONSUMED BY THE ENDEMIC SQUIRREL OF PEROTE \\ (XEROSPERMOPHILUS PEROTENSIS) IN THE PEROTE VALLEY, \\ VERACRUZ, MEXICO
}

\section{Alberto GONZÁleZ-ROMERO,* SANDra MONTERO-BAGATELLA y JesSica DURÁN-ANTONIO}

\begin{abstract}
Instituto de Ecología, A.C., Red de Biología y Conservación de Vertebrados, Carretera Antigua a Coatepec \# 351, El Haya, A.P.63,91070 Xalapa, Veracruz. México <alberto.gonzalez@inecol.mx>, <helena.bagatella@gmail. com>,<jessi_lobo91@hotmail.com>

*Autor para correspondencia: <alberto.gonzalez@inecol.mx>
\end{abstract}

Recibido: 08/08/2017; aceptado: 24/10/2017; publicado en línea: 16/03/2018

Editor responsable: Vinicio Sosa

González-Romero, A., Montero-Bagatella, S. H. y Durán-Antonio, J. (2018) Alimentos consumidos por la ardilla endémica de Perote (Xerospermophilus perotensis) en el Valle de Perote, Veracruz, México. Acta Zoológica Mexicana (n.s.). 34(1), 1-5.

RESUMEN. La ardilla de tierra de Perote (Xerospermophilus perotensis) es un roedor microendémico, clasificado como especie en peligro por la Norma Mexicana NOM-059-SEMARNAT-2010 y la UICN. Los estudios sobre esta especie son escasos y la mayoría de los pocos estudios publicados abordan su distribución y monitoreo poblacional. Mientras que el único estudio previo sobre su dieta, informa solo seis especies de plantas. Basadas en observaciones directas del consumo de alimentos, registramos en nuestro estudio 25 especies de plantas y cuatro fuentes de alimentos animales.

La ardilla terrestre de Perote (Xerospermophilus perotensis Merriam 1893), es una especie de distribución micro endémica de la zona semiárida de la Cuenca de Oriental, la cual abarca partes de los estados de Puebla, Veracruz y Tlaxcala (Best \& Ceballos, 1995; Valdez \& Ceballos, 2005). A pesar de su distribución restringida y de ser una especie considerada por la NOM-059-SEMARNAT-2010 como amenazada (SEMARNAT, 2010; Valdez \& Ceballos, 1997) y como una especie en peligro de extinción por la Unión Internacional para la Conservación de la Naturaleza (IUCN, 2017), son pocos los estudios que se
González-Romero, A., Montero-Bagatella, S. H., \& Durán-Antonio, J. (2018) Food consumed by the endemic squirrel of Perote (Xerospermophilus perotensis) in the Perote Valley, Veracruz, Mexico. Acta Zoológica Mexicana (n.s.). 34(1), 1-5.

ABSTRACT. The Perote ground squirrel (Xerospermophilus perotensis) is a micro-endemic rodent, classified as an endangered species by the Mexican NOM-059-SEMARNAT-2010 and the IUCN. Studies about this species are scarce, with most of the few published studies addressing its distribution and population monitoring. While the unique previous study on its diet, reports only six plant species. Based on direct observations of food consumption, we recorded in our study 25 plant species and four animal sources of food.

han realizado acerca de esta especie, los cuales abordan principalmente su distribución (Best \& Ceballos, 1995; García-Domínguez et al., 2014) y su dinámica poblacional (Castillo-Castillo \& González-Romero, 2010; Ochoa et al., 2012; Ros-Cuellar et al., 2013; Montero-Bagatella \& González-Romero, 2014). Sobre su dieta solamente existe un trabajo (Hernández-Hernández, 2012), quien documenta la dieta de esta ardilla en un sitio particular del Ejido El Frijol Colorado, en el Municipio de Perote, y en donde reporta únicamente seis tipos de alimentos consumidos por esta especie durante una temporada. Debido 
a que el conocimiento de la dieta de Xerospermophilus perotensis es básico para su conservación, particularmente por ser una especie amenazada que los campesinos consideran una plaga, el objetivo de este trabajo fue aumentar el conocimiento sobre los diferentes alimentos que consume esta ardilla mediante la obtención de registros visuales de elementos consumidos por la especie en la zona semiárida del Valle de Perote.

Para registrar el consumo de los diferentes alimentos, durante los recorridos realizados en busca de ardillas, cuando se veía a un animal comiendo, se le observaba por un corto tiempo desde el vehículo con ayuda de binocula-

Cuadro 1. Elementos documentados que consume la ardilla de Perote Xerospermophilus perotensis, mediante observaciones directas en el Valle de Perote, Veracruz. Todo el año se refiere a que la planta es consumida durante la temporada en que la ardilla está activa (abril a noviembre).

\begin{tabular}{|c|c|c|c|c|c|}
\hline \multirow[t]{2}{*}{ FAMILIA } & \multirow[t]{2}{*}{ ESPECIE } & \multicolumn{3}{|c|}{ SITIO } & \multirow[t]{2}{*}{ ÉPOCA DE CONSUMO } \\
\hline & & LLANO & LLANO SALADO & LLANOS MALPAIS & \\
\hline \multicolumn{6}{|c|}{ Materia Vegetal } \\
\hline \multirow[t]{2}{*}{ Asteraceae } & Bidens anthemoides & $\mathrm{X}$ & & & Todo el año \\
\hline & Bidens bipinnatifida & $\mathrm{X}$ & & & Todo el año \\
\hline \multirow{2}{*}{ Cactaceae } & Opuntia robusta & $\mathrm{X}$ & & $\mathrm{X}$ & Verano \\
\hline & Cylindropuntia rosea & & $\mathrm{X}$ & $\mathrm{X}$ & Verano \\
\hline Caryophyllaceae & Cardionema ramosissimum & $\mathrm{X}$ & & & Verano \\
\hline Brassicaceae & Brassica rapa & $\mathrm{X}$ & $\mathrm{X}$ & $\mathrm{X}$ & Otoño \\
\hline \multirow[t]{8}{*}{ Poaceae } & Avena sativa & $\mathrm{X}$ & $X$ & $X$ & Otoño \\
\hline & Bouteloua scorpioides* & $\mathrm{X}$ & $\mathrm{X}$ & $\mathrm{X}$ & Otoño \\
\hline & Hordeum vulgare & $\mathrm{X}$ & $\mathrm{X}$ & $\mathrm{X}$ & Otoño \\
\hline & Muhlenbergia ramulosa & & $\mathrm{X}$ & & Todo el año \\
\hline & Muhlenbergia repens & & $\mathrm{X}$ & $\mathrm{X}$ & Todo el año \\
\hline & Paspalum sp. & $\mathrm{X}$ & & & Todo el año \\
\hline & Scleropogon brevifolius $*$ & $\mathrm{X}$ & & $\mathrm{X}$ & Todo el año \\
\hline & Triticum aestivum & $X$ & $\mathrm{X}$ & & otoño \\
\hline Solanaceae & Physalis orizabae & $\mathrm{X}$ & & $\mathrm{X}$ & Verano \\
\hline Tenebrionidae & Heleodes spp. & $\mathrm{X}$ & & $\mathrm{X}$ & Verano \\
\hline Acrididae & Trimetropis spp. & $\mathrm{X}$ & & $\mathrm{X}$ & Otoño \\
\hline Cricetidae & Peromyscus maniculatus & $\mathrm{X}$ & & & Otoño \\
\hline Carroña** & Varios vertebrados & $\mathrm{X}$ & & & Todo el año \\
\hline Totales & 29 & 23 & 12 & 20 & \\
\hline
\end{tabular}

*Especies reportadas con anterioridad por Hernández-Hernández (2013).

** Incluye a Sceloporus spinosus, Thamnophis sp., Peromyscus sp. y Xerospermophilus perotensis 

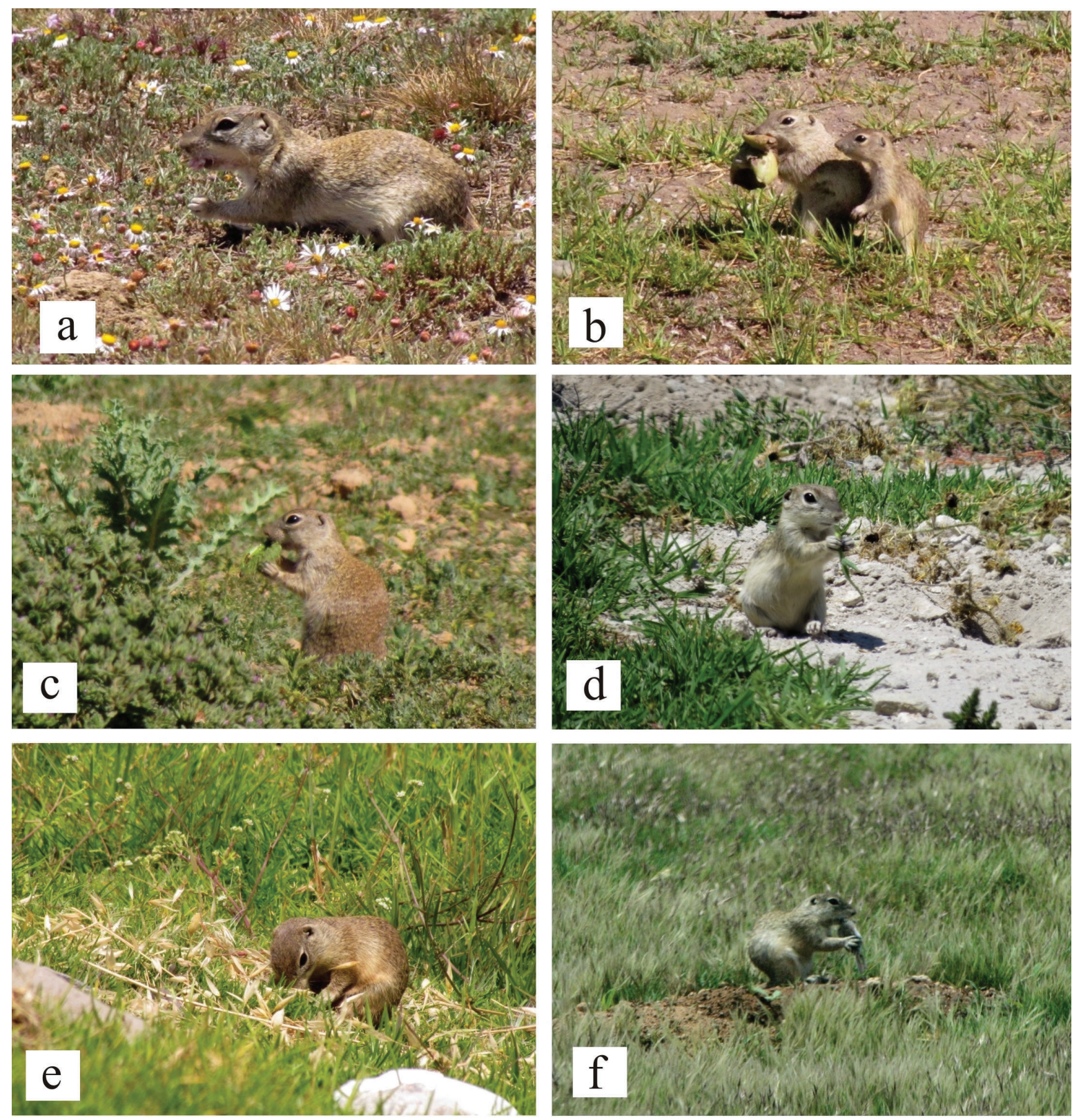

Figura 1. Ardilla de Perote (Xerospermophilus perotensis), también conocida localmente como chichilote o moto, consumiendo diferentes alimentos en el Valle de Perote, Veracruz, México. a) Flores de Erigeron pubescens, b) Fruto de Opuntia huajuaspensis, c) Fruto de Argemone platyceras, d) Porción tierna del tallo de Paspalum sp., e) Granos de Avena sativa, f) Juvenil de Peromyscus maniculatus.

res, de modo que se identificara en forma general lo que estaba consumiendo y se le tomaban fotografías. Luego de manera repentina, alguien bajaba del vehículo y mediante un acercamiento rápido, forzaba a la ardilla a soltar su alimento en el sitio en que lo estaba consumiendo para identificar lo que consumía. Debido a que el área de estudio está fuertemente trasformada por la agricultura, ganadería y extracción de materiales pétreos, no existe una diferenciación marcada de las comunidades vegetales, por lo que los ambientes se clasificaron en las siguientes categorías: 1) Llano: que abarca la mayor parte del Valle, presenta suelos arenosos bien drenados que están dedicados en su gran mayoría a la agricultura y a la expansión urbana, 2) Llano salado: que corresponde a los terrenos altos de la laguna "El Salado", Tepeyahualco, Puebla, con suelos franco-arenosos salinos y 3) Llano malpaís: que 

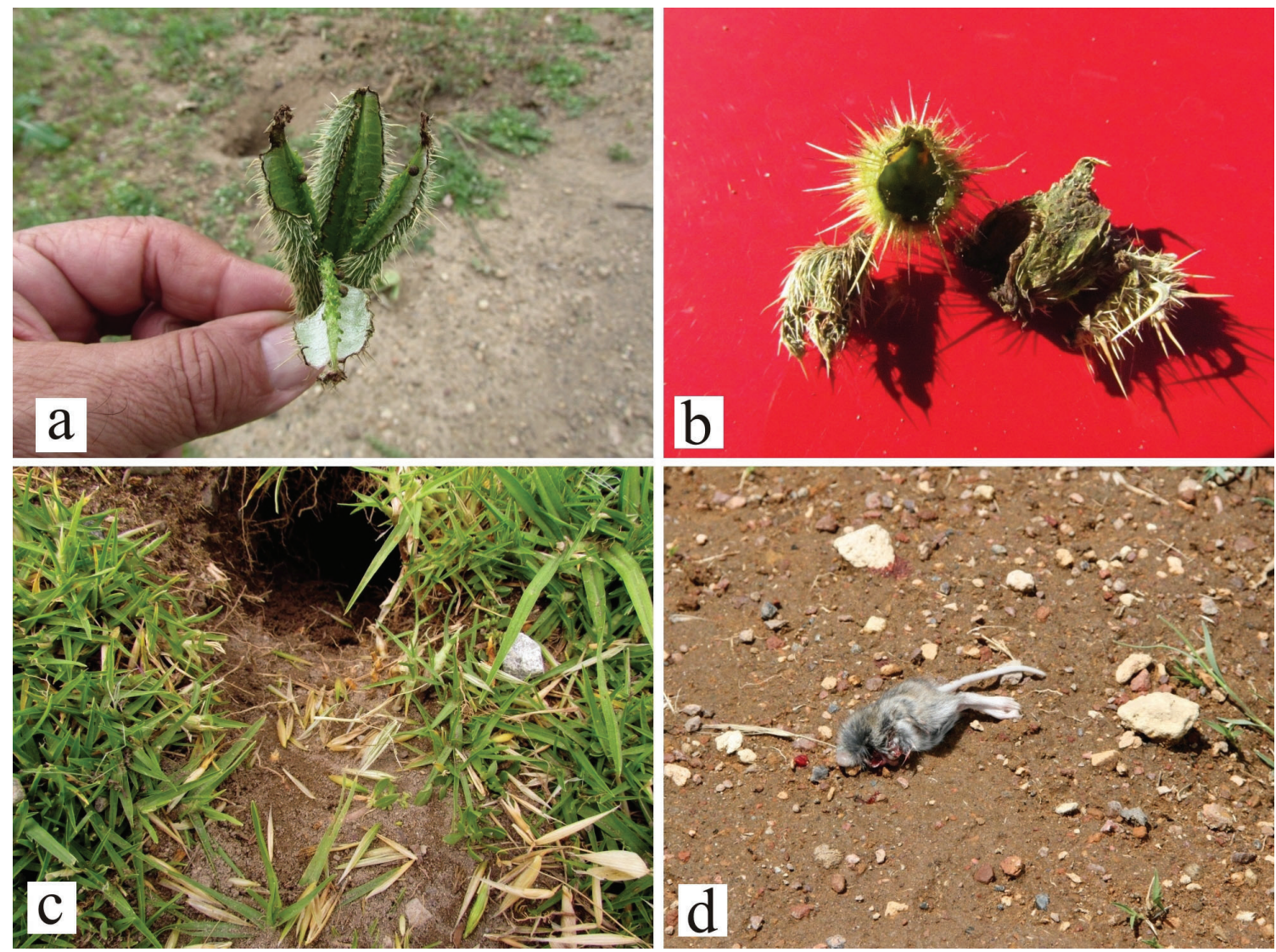

Figura 2. Detalle de cuatro de las especies consumidas por el moto (Xerospermophilus perotensis) en el Valle de Perote, Veracruz, México. a) Fruto de Argemone platyceras, b) Frutos de Solanum rostratum, c) Vainas de los granos de Avena sativa frente a la madriguera de una ardilla,

d) Cuartos inferiores y cola de un juvenil de Peromyscus maniculatus.

son áreas planas en el interior de un derrame basáltico en donde dominan los pastos y la principal actividad es el pastoreo de ganado ovi-caprino.

Se lograron identificar 23 nuevos tipos de alimento en la zona de estudio (Cuadro 1, Figs. 1 y 2), además de los seis que Hernández-Hernández (2012) había registrado. En el Cuadro 1, se tiene la clasificación de los alimentos de acuerdo a su tipo, siendo 25 de origen vegetal y cuatro de origen animal. La familia más consumida fue la Poaceae (pastos) con ocho especies, destacan la avena (Avena sativa), la cebada (Hordeum vulgare) y el trigo (Triticum aestivum), las cuales son de uso agrícola y que la ardilla consume cuando el grano está maduro. Seis especies vegetales son consumidas en las tres áreas estudiadas (Cuadro 1), cuatro de éstas son malezas o plantas ruderales introducidas (Taraxacum officilale, Solanum rostratum, Brassica rapa, Argemone platyceras), que las ardillas están consumiendo con alta frecuencia. MonteroBagatella et al. (2017) identificaron 109 especies vege- tales en el área de estudio, los resultados de este trabajo indican, que de esas especies, 25 son consumidas por la ardilla de Perote en diferentes temporadas.

Dentro de los alimentos de origen animal se registraron tres familias (Cuadro 1). Dos de estas familias, pertenecen a la clase Insecta, la familia Tenebrionidae (coléopteros hediondos), en la cual el género Heleodes es muy abundante en la zona de estudio y la otra familia es la Acrididae, representada principalmente por chapulines del género Trimetropis, abundantes principalmente durante el otoño. La familia Cricetidae, también fue registrada con un ratón ciervo (Figs. 1 y 2). También se registró el consumo de carroña de vertebrados como Sceloporus spinosus, Thamnophis sp., Peromyscus sp. etc. Debido a la frecuencia de observaciones de las ardillas consumiendo carroña en la carretera, es probable que la alimentación de roedores y otros pequeños vertebrados, sea un hecho cotidiano, ya que el tránsito vehicular es constante en el área de estudio y las medidas de paso de fauna son es- 
casas, dejando en riesgo de atropellamiento carretero a los organismos y que especies como las ardillas de Perote aprovechan para alimentarse, aunque se exponen al mismo riesgo de ser atropelladas como efectivamente sucede (González-Gallina et al., 2013). Es importante señalar que se observó a la ardilla de Perote ingiriendo carroña de su propia especie, este comportamiento ya ha sido reportado para otras ardillas e incluso pueden llegar a devorar a las crías de sus homólogos (Torrington et al., 2012).

Aunque este estudio contribuye con los registros de alimentos consumidos por la ardilla, la cual es diversa y varía dependiendo de la estacionalidad, es necesario el realizar un estudio más detallado sobre la dieta de la especie, el cual permita identificar las plantas más importantes para su consumo, la época en que las consume y si los insumos tanto animales como vegetales son utilizados por su disponibilidad, abundancia o si son seleccionados. También es relevante el conocimiento del impacto que tiene el consumo de la ardilla hacia las especies cultivadas, ya que algunos agricultores de la zona las consideran una plaga, provocando un manejo inadecuado de la especie. En este sentido, destacamos que no bastan los estudios de su dinámica poblacional, también es indispensable conocer su dieta, para así tener un mayor conocimiento ecológico, que permita desarrollar un plan de manejo para la especie.

AGRADECIMIENTOS. Reconocemos especialmente a todos los estudiantes que han contribuido con sus tesis al conocimiento de esta especie y por su apoyo en el campo especialmente a Gabriela Mendoza Carreón, Cesia del Carmen Castillo-Castillo, Julia Ros-Cuellar y Alberto González-Gallina. Agradecemos a la Dirección General de Vida Silvestre por los permisos otorgados (SGPA/DGVS/05833/08 y SGPA/DGVS/03369/15), a Granjas Carroll de México, S. de R.L. de C.V. por permitirnos trabajar en sus predios, así como a los ingenieros Tito Tablada, Héctor Flores y Noemí Rosas por el apoyo logístico brindado en el terreno.

\section{LITERATURA CITADA}

Best, T. \& Ceballos, G. (1995) Spermophilus perotensis. Mammalian Species, 507, 1-3

Castillo-Castillo, C. C. \& González-Romero, A. (2010) Áreas de actividad de la ardilla endémica de Perote Xerospermophilus perotensis. Therya, 1, 69-74

García-Domínguez, J. A., Rojas-Soto, O. R., Galindo-González, J. R., González-Romero, A., Pineda-López, M. R. \& Noa-Carranza, J. C. (2014) Present and future potential distribution of the Perote ground squirrel (Xerospermophilus perotensis) under different climatic change scenarios. Mammalia, 78, 507-521.

González-Gallina, A., Benítez-Badillo, G., Rojas-Soto, O. R., \& Hidalgo-Mihart, M. G. (2013) The small, the forgotten and the dead: Highway impact on vertebrates and its implications for mitigation strategies. Biodiversity Conservation, 22, 325-342.

Hernández-Hernández, J. C. (2012) Variación estacional y calidad de la dieta de la ardilla terrestre Xerospermophilus perotensis Merriam 1893 en la localidad el Frijol Colorado, Municipio de Perote, Veracruz. Tesis de Maestría, Instituto de Neuroetología, Universidad Veracruzana, Xalapa, Veracruz.

IUCN. (2017) The IUCN Red List of Threatened Species. Version 2017-1 http://www.iucnredlist.org (accessed on July 07, 2017)

Montero-Bagatella, S. H. \& González-Romero, A. (2014) Análisis demográfico de una especie en estado crítico de conservación: la ardilla de Perote. Therya, 5, 737-750

Montero-Bagatella, S. H., González-Romero, A., Gallina, S., \& Sánchez-Rojas., G. (2017) Relaciones entre las características de la vegetación y las densidades de la ardilla de Perote. Revista Mexicana de Biodiversidad, 88, 691-700.

Ochoa, A., Gasca, J., Ceballos, G. \& Eguiarte, L. (2012) Spatio temporal population genetics of the endangered Perote ground squirrel (Xerospermophilus perotensis) in a fragmented landscape. Journal of Mammalogy, 92, 227-234.

Ros-Cuellar, J., González-Romero, A. \& Flores-Peredo, R. (2013) Parámetros poblacionales del chichilote (Xerospermophilus perotensis Merriam 1893) en el Valle de Perote, Veracruz. Pp.34-39 en: Memorias del XXX Simposio Sobre Fauna Silvestre "Gral. MV. Manuel Cabrera Valtierra. FMVZ-UNAM, México, D.F.

SEMARNAT (Secretaría de Medio Ambiente y Recursos Naturales). (2010) Norma Oficial Mexicana NOM-059-SEMARNAT-2010, Protección ambiental-Especies nativas de México de flora y fauna silvestres-Categorías de riesgo y especificaciones para su inclusión, exclusión o cambio-Lista de especies en riesgo. Diario Oficial de la Federación (segunda sección) México, D.F. 77 pp.

Torrington, R.W. Jr., Koprowski, J. L., Steele, M. A., \& Whatton, J. F. (2012) Squirrels of the world. The Johns Hopkins University Press, Baltimore, E.U.A., 459 pp.

Valdez, M. \& Ceballos, G. (1997) Conservation of endemic mammals of Mexico: the Perote ground squirrel (Spermophilus perotensis). Journal of Mammalogy, 78, 74-82.

Valdez, M. \& Ceballos, G. (2005) Spermophilus perotensis Merriam, 1893. Pp. 568-569 In: Ceballos, G. \& G. Oliva (eds.). Los mamíferos silvestres de México. CONABIO-Fondo de Cultura Económica. México, D.F. 